\title{
USO DO FEROMÔNIO SEXUAL SINTÉTICO PARA CAPTURA DE MACHOS DA TRAÇA-DAS-CRUCÍFERAS' ${ }^{1}$
}

\author{
MIRIAN FERNANDES FURTADO MICHEREFF², EVALDO FERREIRA VILELA ${ }^{3}$, \\ MIGUEL MICHEREFF FILHO ${ }^{4}$ e AGENOR MAFRA-NETO ${ }^{5}$
}

\begin{abstract}
RESUMO - Este trabalho teve como objetivo estudar a atração dos machos da traça-das-crucíferas (Plutella xylostella L., Lepidoptera: Yponomeutidae) por diferentes formulações do feromônio sexual sintético. Os tratamentos consistiram em: 1) formulação comercial; 2) Z-11,16:Ald; 3) Z-11,16:Ac; 4) mistura 7:3 de 2+3;5) mistura 5:5 de 2+3;6) septos de borracha com hexano (testemunha); e 7) cinco fêmeas virgens. A formulação comercial do feromônio sexual sintético propiciou maior captura de machos, não diferindo significativamente de fêmeas virgens e da mistura 5:5 utilizadas como isca. Os componentes isoladamente foram pouco atrativos aos machos. Foram testados cinco modelos de armadilhas. A armadilha Pherocon 1 CP (33,2 machos/armadilha/noite) foi a mais eficiente na captura de machos, seguida pelas armadilhas Cilíndrica Aberta, Cilíndrica Fechada, Delta e Redonda Aberta. Três alturas de instalação da armadilha foram avaliadas. Ocorreu significativamente maior captura de machos na altura do ápice das plantas $(30 \mathrm{~cm}$ do solo). As alturas de 5 e $60 \mathrm{~cm}$ não diferiram entre si.
\end{abstract}

Termos para indexação: Plutella xylostella, manejo integrado de pragas, armadilhas, repolho.

\section{SYNTHETIC SEX PHEROMONE USE FOR FIELD TRAPPING OF DIAMONDBACK MOTH MALES}

\begin{abstract}
The aim of this work was to study the attractiveness of males of Plutella xylostella $\mathrm{L}$. (Lepidoptera: Yponomeutidae) to different formulations of synthetic sex pheromone. The treatments were: 1) commercial formulation; 2) Z-11,16:Ald; 3) Z-11,16:Ac; 4) binary blends 7:3 of 2+3; 5) binary blends $5: 5$ of $2+3 ; 6$ ) rubber septa with hexane (control); 7) five virgin females. The commercial formulation of the synthetic sex pheromone was more attractive to males, and did not differ significantly from virgin females and binary blends 5:5 treatments. The components alone were less attractive to males. Five traps were evaluated: Pherocon 1 CP (wing trap), Delta, "PVC 200", "PVC 250" and Black Round Trap, where the wing trap was more effective in capturing males than the other traps tested. The wing trap was evaluated at 5,30 and $60 \mathrm{~cm}$. More males were caught at $30 \mathrm{~cm}$ above the ground level, while the height of 5 and $60 \mathrm{~cm}$ did not differ significantly.
\end{abstract}

Index terms: Plutella xyslostella, integrated pest management, pheromone traps, cabbages.

${ }^{1}$ Aceito para publicação em 21 de dezembro de 1999. Extraído da monografia de graduação, apresentada pela primeira autora à Universidade Federal de Viçosa (UFV), Viçosa, MG.

${ }^{2}$ Biól., M.Sc., Dep. de Biologia Animal, UFV, CEP 36571-000 Viçosa, MG. E-mail: mirian@alunos.ufv.br

${ }^{3}$ Eng. Agrôn.,Ph.D., Prof. Titular, Dep. de Biologia Animal, UFV, E-mail: evilela@mail.ufv.br

${ }^{4}$ Eng. Agrôn., M.Sc., Dep. de Biologia Animal, UFV. E-mail: mch@alunos.ufv.br

${ }^{5}$ Biól., Ph.D., Pesq. Associado, Dep. of Entomology, University of California, Riverside, USA. E-mail: mafranet@ucrac1.ucr.edu

\section{INTRODUÇÃO}

A traça-das-crucíferas (Plutella xylostella $\mathrm{L}$.) destaca-se como uma das pragas de maior expressão econômica no cultivo de repolho, no Brasil e em várias outras regiões do mundo (Talekar \& Shelton, 1993; Castelo Branco et al., 1996). O uso indiscriminado de inseticidas sem a prévia estimativa dos danos de P. xylostella nos cultivos tem aumentado os custos de produção, eliminado os inimigos naturais e selecionado populações da praga com resistência a diversos inseticidas contendo vários grupos 
de princípios ativos (Zilahi-Balogh et al., 1995; Campos et al., 1997).

Uma das soluções para o problema é o monitoramento populacional de $P$. xylostella, por meio de armadilhas contendo como atrativo o seu feromônio sexual sintético (Baker et al., 1982; Hallett et al., 1995). O monitoramento das mariposas pode fornecer informações indispensáveis para se estimar a população da praga e prever seus danos no cultivo, subsidiando desta forma a tomada de decisão quanto ao emprego de medidas de controle e, muitas vezes, promovendo a redução das pulverizações (Jutsum \& Gordon, 1989).

O feromônio sexual da fêmea de $P$. xylostella foi identificado por Chow et al. (1974). Atualmente, as formulações do feromônio sexual sintético, utilizadas comercialmente para monitoramento, são constituídas por misturas binárias ou terciárias do (Z),11-hexadecenal (Z11-16:Ald), mais o acetato de (Z),11-hexadecenila (Z11-16:Ac) e o (Z)-11-hexadecenol (Z11-16:OH), em proporções variáveis de acordo com a região de ocorrência da espécie (Talekar \& Shelton, 1993).

Variações nas respostas de machos da traça-dascrucíferas foram observadas em Taiwan, Japão, Canadá e Indonésia (Maa, 1986; Zilahi-Balogh et al., 1995), resultantes, provavelmente, de fatores externos, como variações sazonais do meio ambiente ou fatores internos, como diferenças genéticas das populações do inseto. Diferenças na resposta de machos são associadas também às combinações entre componentes e à concentração utilizada (Maa, 1986; Talekar \& Shelton, 1993).

Embora formulações do feromônio sexual sintético sejam comercializadas no Brasil, ainda não existem informações a respeito da atratividade desses produtos sobre machos de populações existentes no país.

O presente trabalho teve como objetivo estudar a atração dos machos de $P$. xylostella por diferentes formulações do feromônio sexual sintético.

\section{MATERIAL E MÉTODOS}

Adultos de P. xylostella foram, inicialmente, obtidos a partir de lagartas e pupas coletadas em cultivos de brássicas, da Universidade Federal de Viçosa, MG.
A criação foi mantida no Laboratório de Feromônios e Comportamento do BIOAGRO/UFV, com dieta à base de folhas frescas de couve comum (Brassica oleracea $\mathrm{L}$. var. acephala D.C.), sob regime de $14 \mathrm{~L} / 10 \mathrm{E}$, a $23 \pm 1^{\circ} \mathrm{C}$ e $70 \pm 2 \%$ de umidade relativa.

Os experimentos foram conduzidos em cultivos comerciais do repolho híbrido AG Master 259 (Agroceres), na Fazenda dos Lemos, Viçosa, MG. As temperaturas máxima e mínima diárias, bem como a umidade relativa do ar, foram fornecidas pela Estação Meteorológica da UFV.

\section{Atratividade das formulações do feromônio sexual sin- tético no campo}

O experimento foi conduzido em cultivo de repolho com plantas de $35 \mathrm{~cm}$ de altura e no estádio de formação da cabeça. Foi avaliada a atração sexual dos machos da traça-das-crucíferas pelos seguintes tratamentos: 1) formulação comercial (IPM Technologies, Oregon, USA); 2) Z11-16:Ald; 3) Z11-16:Ac; 4) mistura 7:3 ( $\mu \mathrm{g} / \mu \mathrm{g})$ de Z11-16:Ald e Z11-16:Ac; 5) mistura 5:5 ( $\mu \mathrm{g} / \mu \mathrm{g})$ de Z11-16:Ald e Z11-16:Ac; 6) somente solvente hexano bidestilado (testemunha), e 7) cinco fêmeas virgens, trocadas a cada dois dias. A formulação comercial utilizada consistiu em uma mistura de 5:5:0,1 dos componentes Z-11,16:Ald, Z-11,16:Ac e Z-11,16:OH, respectivamente.

As armadilhas aladas, comumente utilizadas no monitoramento deste inseto em outros países (Baker et al., 1982), receberam septos de borracha, impregnados com os respectivos tratamentos, na dose de $100 \mu \mathrm{g}$. Foram utilizados três canteiros de repolho, correspondendo a uma área total de $5.600 \mathrm{~m}^{2}$. Cada canteiro representou um bloco, onde sete armadilhas foram arranjadas em duas linhas dispostas transversalmente ao vento e distanciadas em $10 \mathrm{~m}$. Na linha, as armadilhas foram instaladas na altura de $30 \mathrm{~cm}$ e distanciadas em $6 \mathrm{~m}$. As misturas foram definidas com base nas proporções indicadas na literatura para espécies semelhantes, sendo os septos fixados por um arame a $2 \mathrm{~cm}$ da base inferior da armadilha e trocados a cada sete dias.

A contagem e retirada dos insetos capturados foram realizadas após as $8 \mathrm{~h}$, ante o fato de que a ocorrência dos acasalamentos é entre uma hora e seis horas após o início da escotofase (Yamanda \& Koshihara, 1980), recasualizando-se, posteriormente, a localização das armadilhas. As avaliações foram efetuadas a cada 48 horas durante 16 dias consecutivos, e o delineamento experimental foi em blocos casualizados, com três repetições, sendo cada repetição constituída por uma armadilha. A temperatura média diária, no período, foi de $17,4 \pm 0,2^{\circ} \mathrm{C}$, e a umidade relativa $77,8 \pm 0,9 \%$ 


\section{Modelos de armadilha}

Em cultivo de repolho com plantas de $35 \mathrm{~cm}$ de altura e no estádio de formação da cabeça, foi avaliada a eficiência de captura de cinco tipos de armadilhas, sendo duas comerciais: Pherocon $1 \mathrm{CP}$ (armadilha do tipo alada), e Delta e três modelos artesanais: Cilíndrica Fechada e Cilíndrica Aberta, feitas de tubo de PVC de $25 \mathrm{~cm}$ de comprimento e 200 e $250 \mathrm{~mm}$ de diâmetro, respectivamente, e a Redonda Aberta, feita com dois pratos pretos de xaxim unidos por arames, sendo o prato superior de $20 \mathrm{~cm}$, e o inferior, de $32 \mathrm{~cm}$ de diâmetro, e a distância de separação entre os pratos, de $5 \mathrm{~cm}$ (Uchoa-Fernandes \& Vilela, 1994).

Utilizou-se uma área cultivada com repolho e adotouse o mesmo procedimento para a disposição das armadilhas do experimento anterior. $\mathrm{O}$ delineamento experimental foi em blocos casualizados, com três repetições por tratamento, recebendo, cada armadilha, um septo de borracha impregnado com a formulação comercial avaliada anteriormente. A captura de machos foi avaliada a cada 48 horas, durante sete dias consecutivos. A temperatura média, no período, foi de $17,8 \pm 0,4^{\circ} \mathrm{C}$, e a umidade relativa foi de $71 \pm 1 \%$.

\section{Altura de instalação das armadilhas}

O experimento foi conduzido em cultivo de repolho com plantas de $30 \mathrm{~cm}$ de altura e no estádio de pré-formação da cabeça. Foram avaliadas três alturas de instalação de armadilhas: $5 \mathrm{~cm}$ (nível do solo), $30 \mathrm{~cm}$ (ápice das plantas) e $60 \mathrm{~cm}$. Utilizaram-se três canteiros de repolho, cada qual representando um bloco de, aproximadamente, $450 \mathrm{~m}^{2}$, onde três armadilhas Pherocon $1 \mathrm{CP}$, com septo contendo a formulação comercial, foram espaçadas em $10 \mathrm{~m}$ e arranjadas em linha disposta no centro do cultivo.

As avaliações foram realizadas a cada 48 horas durante sete dias consecutivos. O delineamento experimental foi em blocos casualizados, com três repetições por tratamento, sendo cada repetição representada por uma armadilha. A temperatura média diária, no período, foi de $21,3 \pm 0,4^{\circ} \mathrm{C}$ e a umidade relativa, $75 \pm 2 \%$.

Os dados sobre captura de machos por armadilha, por noite, foram analisados quanto à normalidade pelo teste de Lilliefors e submetidos à análise de variância (ANOVA) e separação de médias pelo teste de Tukey $(\mathrm{p}<0,05)$.

\section{RESULTADOS E DISCUSSÃO}

\section{Atratividade das formulações do feromônio sexual sintético}

Os resultados demonstraram diferença significativa na atratividade de machos de $P$. xylostella entre os atraentes utilizados nas armadilhas $(\mathrm{p}<0,05)$. Os componentes, Z-11,16:Ald e Z-11,16:Ac isolados, exerceram baixa atratividade sobre os machos de P. xylostella, em relação as suas referidas misturas (Fig. 1), confirmando, assim, a importância da natureza multicomponente do feromônio sexual sintético para esta espécie, como relatada por Zilahi-Balogh et al. (1995). A necessidade de vários componentes químicos para a eficiência do feromônio sexual sintético tem sido encontrada na maioria dos lepidópteros, a exemplo de Grapholita molesta (Busk) (Tortricidae) e Trichoplusia ni (Hüb.) (Noctuidae) (Linn Junior et al., 1986, 1987). Em contraposição, existem algumas espécies de mariposas cujos machos respondem efetivamente ao feromônio sexual sintético constituído por um único componente, como são os casos de Cydia nigricana (F.) (Tortricidae) e Lymantria dispar (L.) (Lymantriidae) (Bierl et al., 1970; Greenway, 1984).

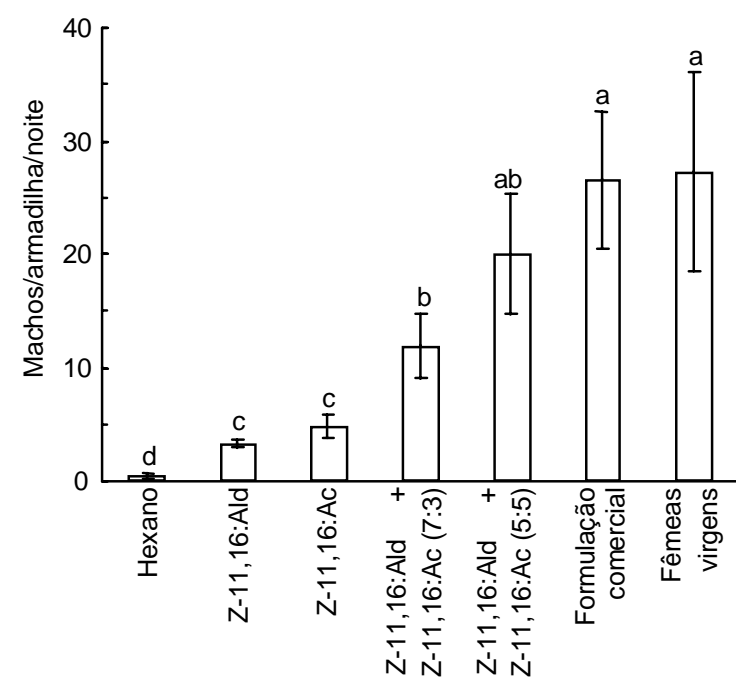

FIG. 1. Número médio ( \pm erro-padrão) de machos de Plutella xylostella capturados por armadilha e por noite, em função do atraente utilizado. Armadilhas com septo de borracha vermelho, impregnado com $100 \mu \mathrm{g}$ do atraente. Valores dentro de parênteses correspondem à proporção entre os componentes da mistura. Avaliação a cada 48 horas, durante 16 dias $(n=28$ armadilhas). Dados transformados em $\log (x+1)$. Médias com a mesma letra não diferem entre si, pelo teste de Tukey $(p>0,05)$. 
A captura dos machos de $P$. xylostella em armadilhas contendo a mistura dos componentes na proporção 5:5 (Z-11,16:Ald : Z-11,16:Ac) não diferiu estatisticamente da obtida pela formulação comercial ou pelas fêmeas virgens. Os resultados mostram uma tendência positiva entre incremento da concentração de acetato na isca e o aumento da captura de machos. Houve maior captura quando o acetato representava $50 \%$ da mistura binária, em relação a $30 \%$. Além disso, segundo Maa (1986), a presença de 80\% a $90 \%$ de aldeído pode inibir o comportamento sexual dos machos de P. xylostella, sendo, portanto, uma das possíveis causas para a menor captura nas armadilhas que continham septo impregnado somente com o aldeído (100\% da composição).

As maiores capturas dos machos foram obtidas com a formulação comercial do feromônio sexual sintético e com as fêmeas virgens, que não diferiram estatisticamente entre si, sendo seguidos pelos demais tratamentos (Fig. 1). Este fato revela o alto potencial de atração da mistura empregada na formulação comercial, sendo competitiva com o feromônio sexual emitido pelas fêmeas no campo. Resultados semelhantes foram obtidos por Ishii et al. (1981), no Japão, e por Chow et al. (1984) em Taiwan. No presente estudo, a maior captura conferida pela formulação comercial em relação à mistura 5:5 de Z-11,16:Ald e Z-11,16:Ac pode ser atribuída à presença do álcool $(Z-11,16: 0 H)$ adicionado à mesma. Koshihara \& Yamanda (1980) relatam que armadilhas com septo na dose de $100 \mu \mathrm{g}$ da mistura de Z-11,16:Ald com Z-11,16:Ac (proporção 1:1) e mais 1\% do álcool Z-11,16:OH, mostrou-se consideravelmente mais atrativa do que uma armadilha com cinco fêmeas virgens.

Segundo Talekar \& Shelton (1993), diferenças na resposta dos machos de P. xylostella ao feromônio sintético podem estar associadas principalmente ao atraente, tanto em termos de mistura entre componentes, como a concentração utilizada. No Japão, elevada captura de machos de $P$. xylostella no campo foi obtida com $100 \mu \mathrm{g}$ do feromônio sexual sintético na primavera e início do verão, enquanto no inverno foi necessário 1,0 mg do produto sintético (Yamanda \& Koshihara, 1980). Contudo, $100 \mu \mathrm{g}$ de feromônio foi suficiente para o armadilhamento de machos de P. xylostella no Canadá (Chisholm et al.,
1979), enquanto em Taiwan foram necessários apenas $50 \mu \mathrm{g}$ de feromônio (Lin \& Chow, 1983).

Estas variações tanto podem ser explicadas pela variabilidade genética, possivelmente por causa do isolamento geográfico das populações, bem como pelos fatores que afetam a resposta dos machos, tais como temperatura e umidade, planta hospedeira, idade das fêmeas e status reprodutivo (McNeil, 1991). Variações similares nas respostas dos machos entre populações isoladas geograficamente, ocorrem em outros lepidópteros e em alguns coleópteros (Cardé et al., 1978).

Embora existam diferenças nas respostas dos machos de P. xylostella ao feromônio sexual sintético em várias partes do mundo, a formulação americana empregada neste trabalho mostrou alto potencial de uso para o monitoramento e controle da referida praga, visto que sua atratividade foi semelhante à das fêmeas virgens. Além disso, o contraste com a pequena captura no tratamento testemunha revelou a ação efetiva do feromônio sexual sintético, contido na formulação comercial, sobre machos de P. xylostella, na região de Viçosa.

\section{Modelos de armadilha}

Houve diferença na captura dos machos entre as armadilhas testadas $(\mathrm{p}<0,05)$. A armadilha Pherocon $1 \mathrm{CP}$ (estilo alada) capturou um número significativamente maior de machos (33,2 machos/ armadilha/noite) em relação às demais, enquanto a Cilíndrica Fechada (5,1 machos/armadilha/noite) e a Cilíndrica Aberta (7,4 machos/armadilha/noite) capturaram os insetos em valores intermediários. As armadilhas Delta (1,7 machos/armadilha/noite) e Redonda Aberta (1,4 machos/armadilha/noite) não diferiram entre si e demonstraram ser muito pouco eficientes na captura dos machos de P. xylostella (Fig. 2).

O formato da armadilha é fundamental para a captura efetiva de machos, influenciando a habilidade de recrutamento da pluma de feromônio e possibilitando que os insetos tenham maior acesso à entrada da armadilha. Murhead-Thomson (1991) relata que o rastro do feromônio pode ser facilmente interrompido, dependendo do tipo de armadilha, afetando o vôo dos insetos para a armadilha. Assim, a eficiência de captura da armadilha está relacionada ao conjun- 
to de suas características, como formato, superfície de adesão, modo de liberação da pluma, e algumas vezes, a cor.

O melhor desempenho da armadilha Pherocon $1 \mathrm{CP}$ pode estar relacionado as suas características de acesso e aprisionamento de machos. A presença de aberturas em todas as direções das armadilhas aladas (multidirecionais) pode ter tido dois efeitos relevantes: a) minimizar a formação errática da pluma de feromônio em razão da disposição da armadilha no campo e da direção do vento; as armadilhas unidirecionais (duas ou poucas aberturas), a exemplo da Delta e Cilíndrica Fechada, exigem a sua adequada instalação em relação ao vento dominante e b) facilitar a entrada dos machos de $P$. xylostella oriundos dos arredores, que comumente não estão em vôo orientado. Além disso, as armadilhas aladas, como a Pherocon $1 \mathrm{CP}$, também possuem um sistema de anteparo que dificulta a saída dos machos que foram atraídos à fonte de feromônio. Os machos, ao tentarem continuar o vôo dentro da

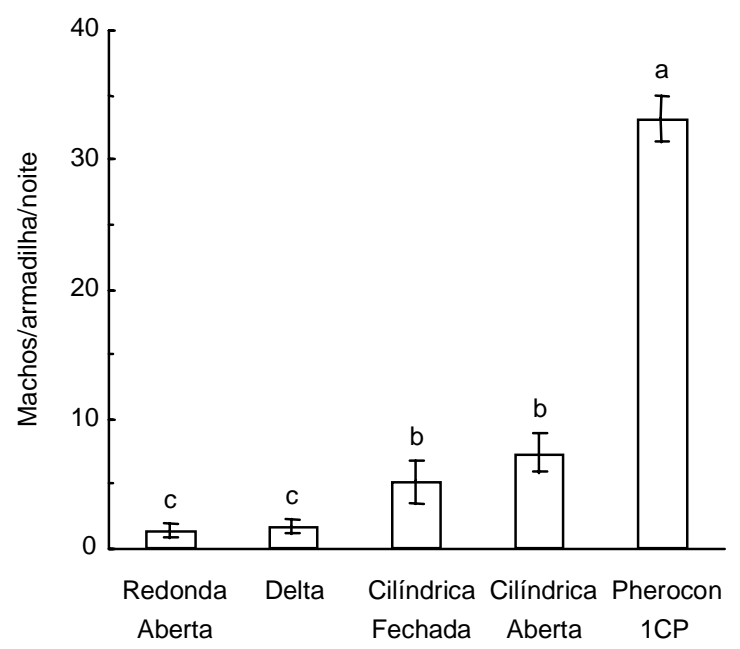

FIG. 2. Número médio ( \pm erro-padrão) de machos de Plutella xylostella capturados por diferentes modelos de armadilhas. Armadilhas com septo de borracha vermelho, impregnado com $100 \mu \mathrm{g}$ da formulação comercial. Avaliação a cada 48 horas, durante 7 dias ( $n=15$ armadilhas). Dados transformados em $\log (x+1)$. Médias seguidas com a letra não diferem entre si, pelo teste de Tukey $(p>0,05)$. armadilha, acabam por bater nos anteparos, caindo sobre o cartão adesivo. A ausência de anteparos pode explicar o resultado obtido com as armadilhas Redonda Aberta, Cilíndricas Aberta e Fechada, pois mesmo possuindo aberturas largas, não foram eficientes na captura de machos, possivelmente por permitirem a fuga dos insetos. Ranga Rao et al. (1991), realizando testes de armadilhas com feromônios para captura de Spodoptera litura F. (Lepidoptera: Noctuidae), concluíram que armadilhas com maior número de aberturas não são necessariamente as mais eficientes para esta praga. No mesmo sentido, Nandagopal \& Soni (1993) relatam que pode ocorrer decréscimo na captura de machos de Aproaerema modicella Deventer (Lepidoptera: Gelechiidae) em armadilhas com ausência de barreiras físicas, em virtude do escape e da grande habilidade de vôo dos insetos.

Vale ressaltar que em todos os modelos foi capturada pequena quantidade de machos, sugerindo a existência de baixa densidade populacional da praga no campo. Mesmo nessa condição o modelo alada propiciou capturas substanciais, demonstrando sua compatibilidade para monitoramento da praga em nossas condições.

\section{Altura de instalação das armadilhas}

As armadilhas instaladas a $30 \mathrm{~cm}$ do solo capturaram significativamente $(\mathrm{p}<0,05)$ maior número de machos (39,4 machos/armadilha/noite), enquanto não houve diferença significativa de captura entre as alturas de $5 \mathrm{~cm}$ (21,0 machos/armadilha/noite) e $60 \mathrm{~cm}$ (19,3 machos/armadilha/noite) (Fig. 3). A altura correspondente ao ápice das plantas provavelmente está associada ao sítio mais conveniente para o chamamento das fêmeas e à altura de vôo dos adultos. De forma semelhante, Reddy \& Urs (1996) verificaram que a melhor altura de instalação da armadilha foi a $30 \mathrm{~cm}$ do solo, permitindo considerável captura de machos de P. xylostella.

Valles et al. (1991) verificaram que armadilhas instaladas no ápice de plantas de abobrinhas foram mais eficientes na captura de machos de Diaphania nitidalis Cramer (Lepidoptera: Pyralidae), comparada às armadilhas instaladas em alturas superiores ou inferiores à região do dossel, demonstrando, assim, a importância da altura de instalação das armadilhas 
empregando fêmeas virgens ou feromônio sexual sintético, no monitoramento de pragas.

A baixa captura dos machos de P. xylostella em armadilhas instaladas a $60 \mathrm{~cm}$ do solo pode ter ocorrido pelo fato de a capacidade intrínseca de vôo do inseto ser baixa, sendo pouco hábil em seguir a pluma formada em alturas mais elevadas. Resultados semelhantes foram obtidos por Chisholm et al. (1979), os quais relatam que $30 \mathrm{~cm}$ seria a altura conveniente, uma vez que a traça-das-crucíferas possui um vôo baixo, preferindo buscar o abrigo conferido pela cobertura da folhagem.

Embora as alturas acima do ápice das plantas possam contribuir para melhor distribuição e alcance da pluma de feromônio no cultivo, a velocidade do vento provavelmente mostrou-se como fator influente sobre a captura de machos a $60 \mathrm{~cm}$ do solo, já que à medida que aumenta a altura este elemento climático se torna mais intenso, dificultando, assim, a movimentação e orientação dos insetos.

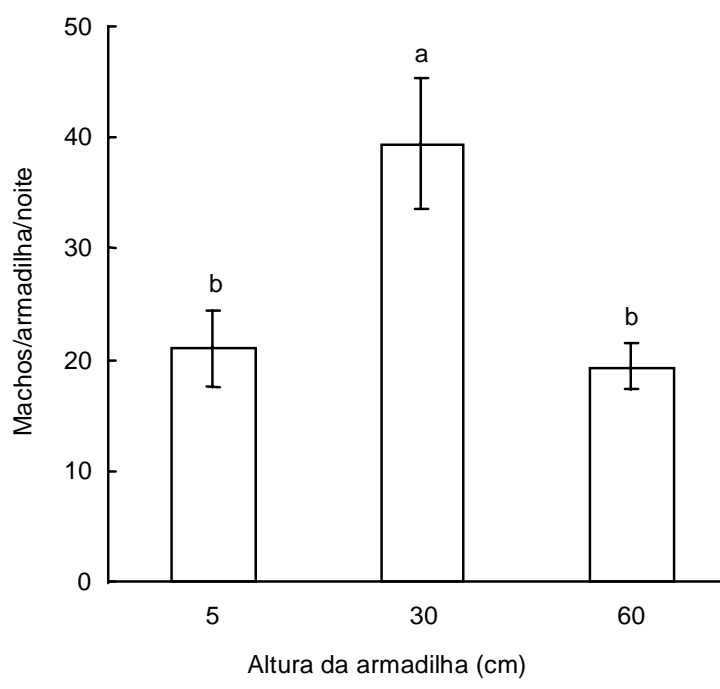

FIG. 3. Número médio ( \pm erro-padrão) de machos de Plutella xylostella capturados em diferentes alturas de instalação das armadilhas. Armadilhas com septo de borracha vermelho, impregnado com $100 \mu \mathrm{g}$ da formulação comercial. Avaliação a cada 48 horas, durante 7 dias $(n=9$ armadilhas). Dados transformados em $\log (x+1)$. Médias seguidas com a mesma letra não diferem entre si, pelo teste de Tukey $(\mathrm{p}>0,05)$.
A baixa captura nas armadilhas instaladas ao nível do solo $(5 \mathrm{~cm})$ pode estar primariamente relacionada com a barreira física gerada pelas plantas, dificultando a dispersão da pluma de feromônio e a orientação dos machos. Outra possibilidade seria o efeito da baixa velocidade do vento, adjacente à superfície do solo e entre as linhas de cultivo, o qual poderia afetar a formação e dispersão da pluma do feromônio.

Para a adoção do sistema de armadilhamento proposto, novos estudos são necessários para que os resultados obtidos sejam confirmados, envolvendo principalmente outras populações de P. xylostella, diferentes condições climáticas e sistemas de cultivo de repolho.

\section{CONCLUSÕES}

1. Para as nossas condições, a formulação de feromônio sexual sintético com proporções iguais entre Z-11,16:Ald e Z-11,16:Ac e com a presença de $Z-11,16: \mathrm{OH}$ é a mais apropriada para a captura de machos de $P$. xylostella, visando o monitoramento populacional.

2. Armadilhas do estilo alada (wing-trap), com abertura de acesso para todas as direções e com anteparos internos, proporcionam a maior captura de machos de P. xylostella.

3. A melhor altura de instalação da armadilha corresponde ao ápice das plantas de repolho.

4. Armadilhas e formulações de feromônio sexual sintético disponíveis no mercado internacional e com as características aprovadas neste trabalho, mostram-se promissoras para o monitoramento de P. xylostella em cultivos de repolho nas condições brasileiras.

\section{AGRADECIMENTOS}

À entidade financeira FAPEMIG, pelo fornecimento da bolsa de pesquisa; ao professor Eraldo Rodrigues de Lima, pelas sugestões dadas durante a elaboração do trabalho.

\section{REFERÊNCIAS}

BAKER, P.B.; SHELTON, A.M.; ANDALORO, J.T. Monitoring of diamondback moth (Lepidoptera: 
Yponomeutidae) in cabbage with pheromones Journal of Economic Entomology, Lanham, v.75, n.6, p.1025-1028, 1982.

BIERL, B.A.; BEROZA, M.; COLLIER, C.W. Potent sex attractant of the gypsy moth: its isolation, identification, and synthesis. Science, Washington, v. 170, p. $87-89,1970$.

CAMPOS, L.C.A.; CASTELO BRANCO, M.; JUNQUEIRA, A.M.R. Suscetibilidade de três populações de traça-das-crucíferas a Bacillus thuringiensis. Horticultura Brasileira, Brasília, v.15, n.1, p.40-42, 1997.

CARDÉ, R.T.; ROELOFS, W.L.; HARRISON, R.G.; VAWTER, A.T.; BRUSSARD, P.F.; MUTUURA, A.; MUNROE, E. European corn borer: pheromone polymorphism or sibling species? Science, Washington, v.199, p.555-556, 1978.

CASTELO BRANCO, M.; VILLAS BÔAS, G.L.; FRANÇA, F.H. Nível de dano de traça-das-crucíferas em repolho. Horticultura Brasileira, Brasília, v.14, n.2, p.154-157, 1996.

CHISHOLM, M.D.; UNDERHILL, E.W.; STECK, W.F. Field trapping of the diamondback moth Plutella xylostella using synthetic sex attractants. Environmental Entomology, Laham, v.8, n.3, p.516$518,1979$.

CHOW, Y.S.; CHIU, S.C.; CHIEN, C.C. Demonstration of a sex pheromone of the diamondback moth (Lepidoptera: Plutellidae). Annals of Entomological Society of America, Laham, v.67, n.3, p.510-519, 1974.

CHOW, Y.S.; LIN, Y.M.; LEE, N.S.; TENG, H.J. The disruption effect of the synthetic sex pheromone and its analogues on diamondback moth, Plutella xylostella L. in the field. Academia Sinica Institute of Zoology Bulletin, Taipei, v.23, n.2, p.119-122, 1984.

GREENWAY, A.R. Sex pheromone of the pea moth, Cydia nigricana F. (Lepidoptera: Olethreutidae). Journal of Chemical Ecology, New York, v.19, n. 4, p.973982, 1984

HALLETT, R.H.; ANGERILLI, N.P.D.; BORDEN, J.H. Potential for a sticky trap monitoring system for the diamondback moth (Lepidoptera: Yponomeutidae) on cabbages in Indonesia. International Journal of
Pest Management, London, v.41, n.4, p. 205-207, 1995.

ISHII, T.; NAKAMURA, K.; KAWASAKI, I.; NEMOTO, H.; TAKAHASHI, K.; KUBOTA, A. Active space of the sex pheromone of diamondback moth, Plutella xylostella L. (Lepidoptera: Yponomeutidae). Japanese Journal of Applied Entomology and Zoology, Tokyo, v.25, n.2, p.71-76, 1981.

JUTSUM, A.R.; GORDON, R.F.S. Pheromones: importance to insects and role in pest management, In: JUTSUM, A R.; GORDON, R.F.S. (Ed.). Insect pheromones in plant protection. New York : J. Wiley, 1989. p.1-16.

KOSHIHARA, T.; YAMANDA, H. Attractant activity of the female sex pheromone of diamondback moth, Plutella xylostella L. and analogue. Japanese Journal of Applied Entomology and Zoology, Tokyo, v.24, n.1, p.6-12, 1980.

LIN, Y.M.; CHOW, Y.S. Field evaluation and stability studies of the synthetic pheromone of the diamomdback moth, Plutella xylostella. In: $\mathrm{CHOU}$, C.H.; WALLER, G.R. (Ed.). Allelochemicals and pheromones. Taipei : Academia Sinica-Taiwan Institute of Botany, 1983. p.147-155.

LINN JUNIOR, C.E.; CAMPBELL, M.G.; ROELOFS, W.L. Male moth sensitivity to multicomponent pheromones: critical role of female-released blend in determining the functional role of components and active space of the pheromone. Journal of Chemical Ecology, New York, v.12, n.3, p.659-668, 1986.

LINN JUNIOR, C.E.; CAMPBELL, M.G.; ROELOFS, W.L. Pheromone components and active spaces: what do moths smell and where do they smell it? Science, Washington, v.237, p.650-652, 1987.

MAA, C.J.W. Ecological approach to male diamondback moth response to sex pheromone. In: DIAMONDBACK MOTH MANAGEMENT INTERNATIONAL WORKSHOP, 1., Tainan. Proceedings. Tainan : Asian VegeTable Research and Development Center, 1986. p.109-123.

McNEIL, J.N. Behavioral ecology of pheromone-mediated communication in moths and its importance in the use of pheromone traps. Annual Review of Entomology, Palo Alto, v.36, p.407-430, 1991.

MURHEAD-THOMSON, R.C. Trap responses of flying insects. In: MURHEAD-THOMSON, R.C. (Ed.).

Pesq. agropec. bras., Brasília, v.35, n.10, p.1919-1926, out. 2000 
Insect behavior. London : Academic, 1991. p.152179.

NANDAGOPAL, V.; SONI, V.C. Evaluation of pheromone traps for groundnut leaf-miner Aproaerema modicella. Indian Journal of Agricultural Sciences, New Delhi, v.63, n.1, p.64-67, 1993.

RANGA RAO, G.V.; WIGHTMAN, J.A.; RANGA RAO, D.V. The development of a standard pheromone trapping procedure of Spodoptera litura F. (Lepidoptera: Noctuidae) population in groundnut (Arachis hyopogaea L.) crops. Tropical Pest Management, London, v.37, n.1, p.37-40, 1991.

REDDY, G.V.P.; URS, K.C.D. Studies on the sex pheromone of the diamondback moth Plutella xylostella (Lepidoptera: Yponomeutidae) in India. Bulletin of Entomological Research, Wallingford, v.86, p.585-590, 1996.

TALEKAR, N.S.; SHELTON, A.M. Biology, ecology and management of the diamondback moth. Annual Review of Entomology, Palo Alto, v.38, p.275-301, 1993.
UCHOA-FERNANDES, M.A.; VILELA, E.F. Field trapping the tomato worm Scrobipalpuloides absoluta Meyrick (Lepidoptera: Gelechiidae) using virgin females. Anais da Sociedade Entomológica do Brasil, Londrina, v.24, n.1, p.159-164, 1994.

VALLES, S.M.; CAPINERA, J.L.; TEAL, P.E.A. Evaluation of pheromone trap design, height and efficiency for capture of male Diaphania nitidalis (Lepidoptera: Pyralidae) in a field cage. Environmental Entomology, Laham, v.20, n.5, p.1274-1278, 1991.

YAMANDA, H.; KOSHIHARA, T. Flying time of diamondback moth, Plutella xylostella L., to light trap e sex pheromone trap. Japanese Journal of Applied Entomology and Zoology, Tokyo, v.24, n.1, p.30-32, 1980

ZILAHI-BALOGH, G.M.G.; ANGERILLI, N.P.D.; BORDEN, J.H.; MERAY, M.; TULUNG, M.; SEMBEL, D. Regional differences in pheromone response of diamondback moth in Indonesia. International Journal of Pest Management, London, v.41, n.4, p.201-204, 1995. 